\title{
Signal Detection Accuracy of Digital Accelerometers for Ballistocardiographic Propose
}

\author{
Nico Jähne-Raden, Klaus-Hendrik Wolf and Michael Marschollek \\ Peter L. Reichertz Institute for Medical Informatics University of Braunschweig - Institute of \\ Technology and Hannover Medical School, Germany
}

\begin{abstract}
The Ballistocardiography is a method to gain detailed information about cardiac function parameters using heart's transferred movements on the body surface. In our current work, we try to achieve such a precision and sensitivity with digital accelerometers. We created a test set-up under lab conditions in which we used a piezo-vibration generator $(P V G)$ with four times four different high-sensitive triaxial accelerometers on four boards. We used $1 \mathrm{~Hz}$ and $2 \mathrm{~Hz}$ for physiological-like signals, as well as $10 \mathrm{~Hz}$ for comparison proposes. Furthermore, we recorded neutral signals for noise information. All signals were measured by sensor's z-axis. Altogether we got 128 physiological-like-signal data sets. For the analysis, we used the auto-correlation via sliding-window. With this method, we determined the mean periodic intrasignal-correlation, which is used as a base value. Furthermore, we used the cross-correlation (CC) for inter-signal-corr. between similar/diff. sensors, synthetic double-differentiated voltage-like-signals and diff. signal-morphologies (incl. neutral). To conclude, such low-frequency-signals, based on intra-signal periodicity, can be distinguished properly by $C C$. The intra-signal-correlation is on average. 0.73 , values up to 0.98 are reached. In contrast, inter-signal comparisons have correlation of $<0.04$ max (neutral) and 0.22 between comparable signals. Due to the low accel. triangle signals show low correlation, but are clearly distinguishable from noise.
\end{abstract}

\section{Introduction}

Sensor systems have been an established part of medical diagnostics for a long time. In addition to the conventional systems, accelerometers have for some years now increasingly entered the medical research.

However, the use of accelerometers is usually reduced to use of activity determination. With progressing technical advances, digital accelerometers have become growing attractive for medical diagnostics for the investigation of signalmorphological changes in body acceleration, such as for the Ballistocardiography (BCG).

The BCG is a method to gain detailed information of body movements imparted by the ballistic forces associated with cardiac contraction and ejection of blood and with the deceleration of blood flow through the large blood vessels. These heart related movements are translated by a sensor device into an electrical potential, which is suitably amplified and recorded (see [1]).

For the measurement of those rather low accelerated movement signals with tiny amplitudes highly sensitive sensors are needed. With sensor systems, containing such accelerometers, patients can be supported in everyday life after a long confinement to bed, with a myocardial failure or other chronic heart diseases. Within the mobilization process medical professionals can monitor heart function parameters while the patients are in their own homes.

\subsection{Motivation}

Within our BCG project, we are pursuing the aim of utilizing the ballistocardiography as a medical-diagnostic tool for the discrete and long-term ready monitoring of progressive, function limiting heart diseases. With such a device, we will be able to use the BCG as a non-invasive method to gain more information about the heart's function, besides existing tools like ECG. In comparison to the ECG, the BCG collects information of the actual movement of the heart, not only about the electrical excitation, which can look like a healthy signal but hides physical function limitations.

In addition, the use of BCG technology in combination with other non-invasive, diagnostic methods, such as pulse oximetry, can determine the blood pressure over the pulse transfer time (see [2]). This blood pressure determination is furthermore far less disturbing than the conventional measurement by means of a sphygmomanometer and can thus also be used for sleep measurement.

To gain proper accelerometers to create a BCG board ready for the diagnostic use, it has to be determined if low-cost, digital, triaxial acceleration sensors can measure signal with small amplitudes and low vibration frequency, like in heart movement. For this we created the vibration generator trial setup to evaluate the accelerometer functions of basic signal measurement and data accuracy. 


\subsection{Related Work}

The work on the utilization of BCG for medicine and the investigation of information content of ballistocardiographic data, as well as their connection to heart functions is a current international research topic.

Thus, in the past, the methodology that is originated from space exploration was aimed at making "earth" medicine accessible despite to the limitations. This was done, among other things, with first attempts by parabolic flights of an international research group in cooperation with the European Space Agency. There, current technology could be investigated independently of earth's gravitational influences compared to the ECG technology (see [3]).

Various accelerometer sensors have already been used by other international research groups and have also been designed in some cases. These systems include immobile systems, such as weighing scales, as well as portable devices (see [4] [5] [6] [7] [8]).

These devices use analog sensors and are rather unhandy in the implementation. Due to the size of the created devices, some limitations occur with this.

Prior to the creation of a BCG board as a diagnostic tool, various trials and measurements have been done, including in cooperation with the Physikalisch-Technische Bundesanstalt Braunschweig, Germany, to determine what features such a sensor system should have.

At first, various measurements and investigations were carried out to determine the appropriate sensor technology [9]. Furthermore, the selected acceleration sensors were examined for their precision [9].

Also, the suitability for ballistocardiographic measurement was examined. This was done with a series of test subjects using reference measurements using established technologies [9].

\section{Method}

For the testing of the four chosen acceleration sensors (LIS3DSH [STMicroelectronics], BMA280 [Bosch], KX1231039 [Kionix], FXL8471Q [Freescale]) in terms of precision for measuring of small-amplitude, low-frequency movement signals, we created a test set-up under lab conditions in which we used a piezo-vibration generator. Furthermore, we used four boards containing all four different, high-sensitive triaxial accelerometers. Every sensor on the board is equal oriented.

Besides, for the test set-up we putted between ground and array, as well as array and PVG a collisional-buffer-layer. Such buffering layer is analogous to the soft tissue on the human body between heart and sensor like skin.

In figure 1 a model of the PVG trial set-up can be seen. Within this picture the different buffer, as well as the position of the sensor board and PVG are shown.

With this set-up, we measured four different voltage signals (ECG-like, square, sine, triangle) generated by a connected signal-generator, which convert the voltage in a specific oscillation.

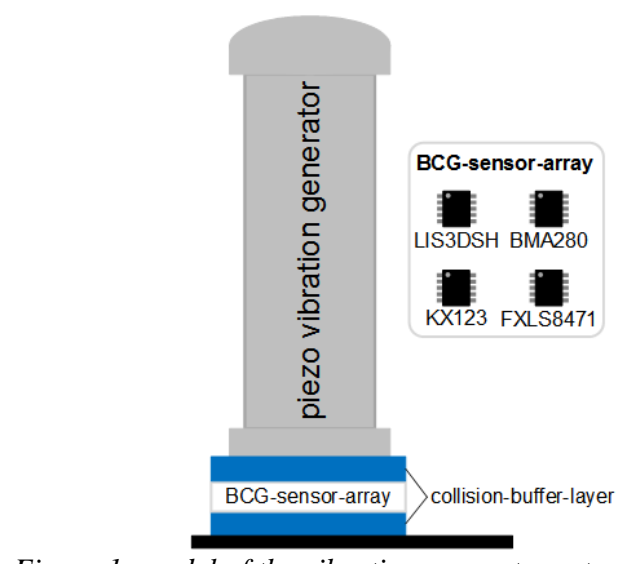

Figure 1: model of the vibration generator set-up including the sensor board

Figure 2 shows up the test set-up including the PVG, the signal generator and the accelerometer board.

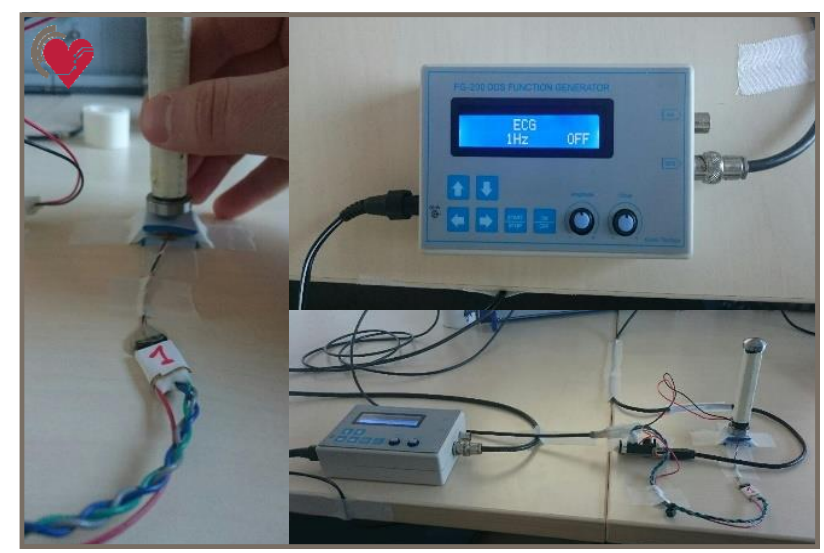

Figure 2: photos of the trial set-up including the signal generator, vibration generator and the sensor board

We used $1 \mathrm{~Hz}$ and $2 \mathrm{~Hz}$ for physiological-like signals, as well as $10 \mathrm{~Hz}$ for comparison proposes. Furthermore, we recorded neutral signals for noise information.

All signals were measured by sensor's z-axis. Altogether we got 128 physiological-like-signal data sets, as well as 16 neutral data sets.

For the analysis, we used the auto-correlation via a 10 -second wide sliding-window, which is putted step-wise for one second. With this method, we determined the mean periodic intrasignal-correlation, which is used as a fundamental value. Furthermore, we used the cross-correlation for inter-signalcorr. between similar and different sensors, synthetic doubledifferentiated voltage-like-signals and different signalmorphologies and the neutral signal.

To conclude, such low-frequency-signals, based on intra-signal periodicity, can be distinguished properly by auto-correlation.

\section{Results}

For the conclusion, several different results can be considered. First the intra-signal cross-correlation results will be shown. For the intra-signal cross-correlation all 128 data samples were 
used, to gain a fundamental value to distinguish with the crosscorrelation result. All measured data sets of the four times four sensors were used by the determined raw data, as well as by low-pass filtered data signal.

In figure 3 the synthetic voltage output signal (in grey) for the four different signals, as well as the measured raw acceleration signal (in blue) from one used FXLS8471 sensor can be seen.

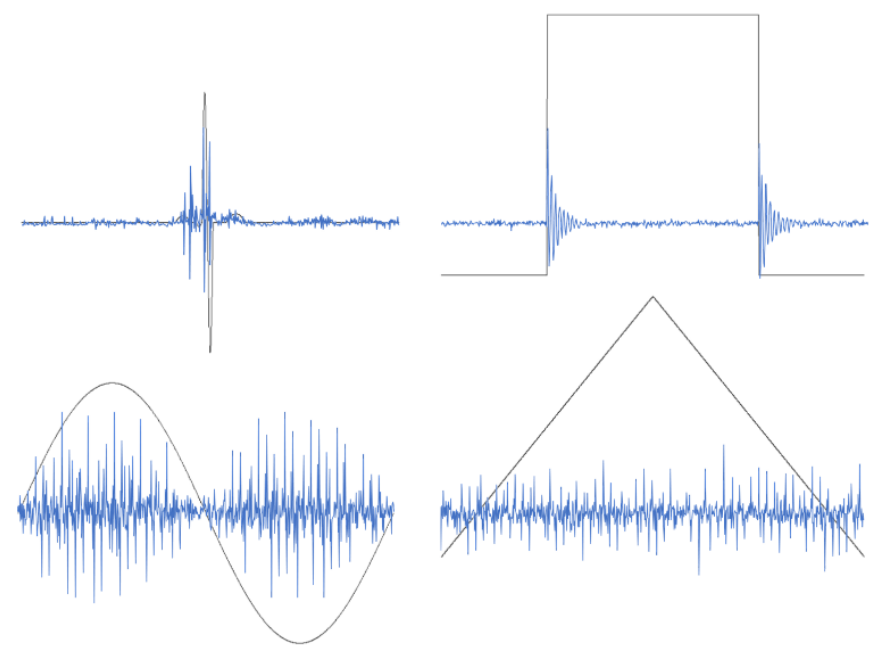

Figure 3: four signal comparisons, between the voltage signal of the signal generator (grey) and the measured acceleration signal (blue)

The unfiltered raw data samples show poor results in terms of the auto-correlation. The intra-signal-correlation for the raw data is on average 0.25 and on maximum about 0.53 . These values are more or less equal within all sixteen sensors, as can be seen by the variance value of 0.01 .

The best results related to the raw data shows the FXLS8471 sensor (Freescale) followed by the KX123-1039 (Kionix).

Focusing on the several signals within the square signal all sensors show the best correlation results followed by sine signal, ECG-like signal and at least the triangle signal. Only the Freescale sensor shows some good correlation results within the triangle signal.

After using the low-pass filter to adjust the data samples, the Kionix shows the best results in terms of correlation. The average low-pass-filtered data correlation for the intra-signal analysis is 0.73 , the maximum is 0.98 . The variance of this consideration is 0.007 .

For the raw acceleration output for the comparison of sensors of the same kind for all four different boards the correlation results of 0.08 on average and on maximum of 0.38 can be determined. Under the use of the low-pass filter a mean correlation result of 0.82 and on maximum of 0.98 can be determined.

Besides we also compared all sensor types, which are 1152 correlations in sum. The raw data correlation by mean is 0.06 and the best is 0.29 . The correlation result using the filtered output data results on average a correlation of 0.82 and on maximum 0.98 . which is equal to the array-wise comparison. In contrast to the intra-signal results and the comparison of the same signals of different sensors (some and different manufacturers), a relatively low inter-signal correlation result is favored to proof a signal difference.

First the correlation results of the comparison with noise signal shall described. The neutral data correlation showed up a correlation of less than 0.04 by maximum for the raw data. Using a low-pass filter the correlation results decreases to 0.00035 , with a variance of 0.0000001 .

For the comparison of all other signal types 192 data correlation were done. For the inter-signal cross-correlation a mean result value of 0.04 can be determined. The maximum result is at 0.22 . In general, it can be said that all except eight data sample show a correlation under 0.1 . The rest seven of eight are correlations of sine and triangle signal including the maximum. The last one is ECG-like and square signal comparison with a result of 0.11 . Considering the comparison measurement at $10 \mathrm{~Hz}$, it can be seen that the noise level within the acceleration output is refined, which can be observed in a decreasing variance. This increase in accuracy can also be observed to a lesser extent with the $2 \mathrm{~Hz}$ measurements.

\section{Conclusion}

Looking at the intra-signal result it can be seen that for a high correlation a proper filtering is needed. This is caused by the noise behavior of the several sensors. The best results show the Freescale, as well as the Kionix sensors. This may is caused by the quite low signal to noise ratio of both components. The better filtering results in KX123-1039 data output is related to the very low noise combined with some occurring artefact peaks. Such peaks are easily and effectively erasable by filtering.

The results of the inter-sensor correlation show quite good outcomes under the restriction of using filtered data, otherwise the output morphology is to different for a high correlation.

How it can be seen in figure 3 the several signals con be easily distinguished by the eye. Furthermore, ECG and square, as well as sine square show some similarities. This may causes the increasing correlation within these data sets. Besides, through the unspecific morphology of the triangle signal it is clearer why a higher correlation occurs. Due to the low acceleration within the triangle no significant acceleration can be detected, but the signal is clearly distinguishable from noise.

As a summary, it can be said, that it is possible to gain a precise acceleration signal from small-amplitude and low-frequency movements. Furthermore, it is also possible to distinguish such signal by its morphologies, even if such signal morphology is similar to others. Caused by its properties a minimum of acceleration is needed. But as can be seen within triangle signal the acceleration can be at a very low value, like caused by vibration friction at the PVG.

Every chosen signal, including the neutral one, can be clearly distinguished from all others. With this information, we can go further with the knowledge that a small amplitude, lowfrequency movement can be measured highly-sensitively. This is a basis for analysis of real physical heart data, pathologic, as well as healthy, to distinguish them.

Moreover, with gained knowledge from the actual work and the 
previous trials and tests, we were able to develop a new highlyspecific BCG board. Figure 4 shows the results of the development process, the new small BCG board.

In the near future, it is planned to discover the properties of the new hardware by repeating the precision trials.

Furthermore, a comprehensive clinical trial is planned to gain information about pathological heart movement and to expand the regular heart movement data amount.

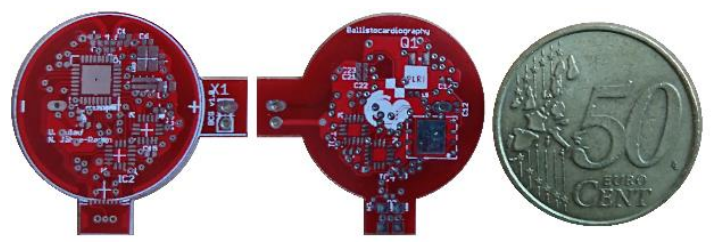

Figure 4: new developed BCG board (top and bottom side) with scale $(0.50 €$ coin)

\section{Limitations}

Due to the technical limitations of the used hardware only one axis at the time could be used to guaranty the needed sample frequency. With new hardware, a second trail using all three axes of the sensor can be realized.

It is not known how accurate the used PVG device is. For the future, the trial can be repeated with the support of the PTB and the use of a laser vibrometer, which can measure the actual PVG movement.

The used synthetic movements are not real heart related movements but in terms of amplitude and frequency similar to those.

\section{Acknowledgements}

We thank the Physikalisch-Technische Bundesanstalt, Braunschweig, Germany - Department of Acoustics and Dynamic subdivision representing of acceleration for the consulting, the allocation of the PTB laboratory and the support at measurement. Besides these supporter, we like to thank Prof. Jens Tank and Dr. med. Karsten Heusser from the Institute of Clinical Pharmacology of the Hannover Medical School,
Germany, for helping in medical related task and questions.

\section{References}

[1] Giovangrandi L, Inan OT, Wiard RM, Etemadi M, Kovacs GTA. Ballistocardiography--a method worth revisiting. Conf Proc IEEE Eng Med Biol Soc 2011; 2011:4279-82.

[2] Kim C, Carek AM, Mukkamala R, Inan OT, Hahn J. Ballistocardiogram as Proximal Timing Reference for Pulse Transit Time Measurement: Potential for Cuffless Blood Pressure Monitoring. IEEE Trans Biomed Eng 2015; 62(11):2657-64.

[3] Deliere Q, Migeotte P, Neyt X, Funtova I, Baevsky RM, Tank J et al. Cardiovascular changes in parabolic flights assessed by ballistocardiography. Conf Proc IEEE Eng Med Biol Soc 2013; 2013:3801-4.

[4] Di Rienzo M, Vaini E, Lombardi P. Wearable monitoring: A project for the unobtrusive investigation of sleep physiology aboard the International Space Station. In: Computing in Cardiology 2015: Volume 42 : September 6-9, 2015, Nice, France. Piscataway, NJ: IEEE; 2015. p. 1258 .

[5] Carek AM, Inan OT. Robust Sensing of Distal Pulse Waveforms on a Modified Weighing Scale for Ubiquitous Pulse Transit Time Measurement. IEEE Trans Biomed Circuits Syst 2017.

[6] Wiens AD, Etemadi M, Roy S, Klein L, Inan OT. Toward continuous, noninvasive assessment of ventricular function and hemodynamics: wearable ballistocardiography. IEEE J Biomed Health Inform 2015; 19(4):1435-42.

[7] Javaid AQ, Ashouri H, Inan OT. Estimating systolic time intervals during walking using wearable ballistocardiography. In: 3rd IEEE EMBS International Conference on Biomedical and Health Informatics. Piscataway, NJ: IEEE; 2016. p. 549-52.

[8] Di Rienzo M, Meriggi P, Vaini E, Castiglioni P, Rizzo F. 24h seismocardiogram monitoring in ambulant subjects. Conf Proc IEEE Eng Med Biol Soc 2012; 2012:5050-3.

[9] Jähne-Raden N, Märtin T, Marschollek M, Heusser K, Tank J. BCGmapping of the thorax using different sensors: First experiences and signal quality. In: IEEE SENSORS 2016: Orlando, Florida, USA, October 30November 2, 2016 : 2016 proceedings papers. Piscataway, NJ: IEEE; 2016. p. 1-3.

[10] Büsching F, Kulau U, Wolf L. Architecture and evaluation of INGA an inexpensive node for general applications. In: 2012 IEEE Sensors. p. 14.

Address for correspondence:

Nico Jähne-Raden

Peter L. Reichertz Institute for Medical Informatics University of Braunschweig - Institute of Technology and Hannover Medical School

Hannover Medical School

Carl-Neuberg-Str. 1

D-30625 Hanover

Nico.Jaehne-Raden@plri.de 